\title{
Massive ovarian oedema: a case report
}

\begin{abstract}
Massive ovarian oedema is a rare entity, non-neoplastic tumor of the ovary. We report a case of massive ovarian oedema (MOO) in a 18-year-old female who presented with abdominal pain along with large solid pelvis mass. It is widely accepted that MOO results from the incomplete torsion of the ovary causing accumulation of fluid within the stroma and enlargement of the ovary. Awareness of this rare lesion due to its lack of pathognomonic features may allow surgeons to a conservative management preventing unnecessary ooohorectomy in young patients.
\end{abstract}

Volume 9 Issue 5 - 2018

\author{
Cecilia Escayola,' Guillermo Ripoll,' Antonio \\ Cardesa, ${ }^{2}$ Juan Jose Torrent ${ }^{3}$ \\ 'Gynecological Surgeon, Hospital el Pilar, Spain \\ ${ }^{2}$ Department of Pathology, Hospital el Pilar, Spain \\ ${ }^{3}$ Gynecological Oncological Surgeon, Hospital el Pilar, Spain
}

Correspondence: Cecilia Escayola MD, Gynecological Surgeon, Hospital el Pilar, Barcelona, Balmes 27I, Spain, Tel 34687439884, Email ceciliaescayola@gmail.com

Received: August 13, 2018 | Published: October 03, 2018
Abbreviations: CEA, carcinoembryonic antigen; MRI, magnetic resonance imaging; MOO, massive ovarian oedema; SST, sclerosing stromal tumour

\section{Introduction}

An 18-year-old unmarried girl was referred to our clinic with selflimited and vague abdominal pain for 3 months with no relieving or aggravating factors. Her menstrual cycles were regular and had rheumatoid arthritis controlled by immunosuppressive diseasemodifying antirheumatic drug.

On physical examination, she had a non-tender mobile adnexal mass measuring $14 \mathrm{~cm}$, extending from the pelvis to the liver. Ultrasound examination revealed an adnexal mass measuring $150 \times 101 \mathrm{~mm}$ with thick septations but no papillary projections. Color Doppler was negative. There was no ascetic fluid seen. The serum levels of Cancer Antigen 153 (CA - 15.3), Carcinoembryonic Antigen (CEA) and Cancer Antigen 19.9 (CA 19.9) were within normal limits. Cancer Antigen $125(\mathrm{CA}-125)$ serum levels were at 71.80. Magnetic resonance imaging (MRI) of the pelvis showed a mass lesion in the left pelvic region, measuring $142 \times 93 \times 125 \mathrm{~mm}$ with multiple thick septations (Figure 1).
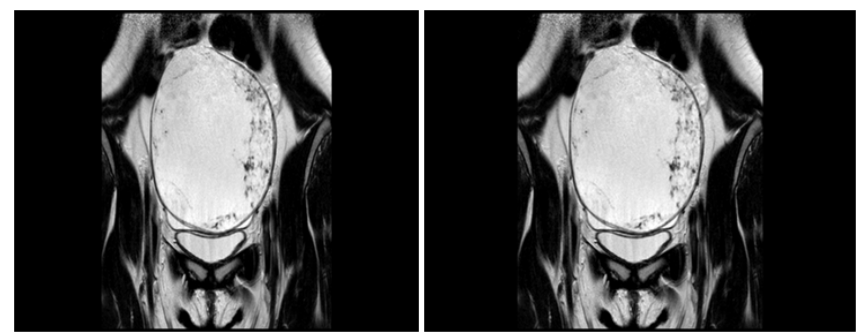

Figure I MRI image of an enlarged left ovary. There are peripheral enlarged follicles.

Based on the above described findings, the lack of guidelines and misleading clinical symptoms, our tumor board decided to offer the patient a laparotomy with left salpingo-oophorectomy. We performed an infraumbilical incision of the abdomen. The right ovary and uterus were macroscopically normal. Gross examination showed a marked left ovarian enlargement with smooth white external surface. The uncertainty of malignant potential due to the size of the lesion was the reason to perform a salpingo-oophorectomy. En bloc resection of the tumor was performed. One litre of clear fluid emerged after the incision of the surface. Microscopic examination revealed marked oedema of ovarian stroma with its architecture preserved. Normal germinal follicles were present without signs of necrosis but abundant cystic lesions were seen on the upper surface of the ovary (Figure 2) (Figure 3).

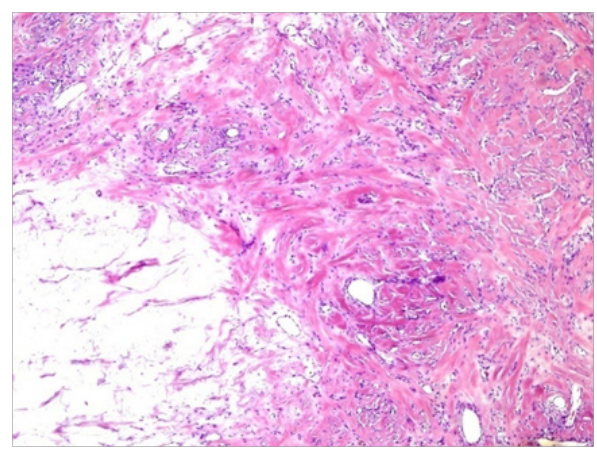

Figure 2 Fibroblastic stroma with lymphatic channels. Stromal oedema on the left.

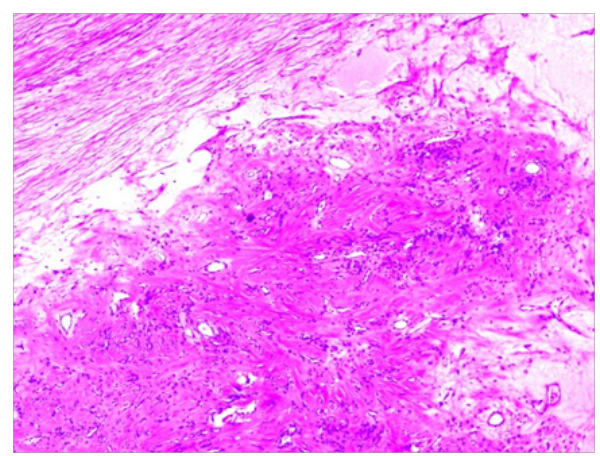

Figure 3 Central region of fibrosis with vascular and lymphatic channels. Cortex with oedematous infiltration on the left.

Following surgery, the patient showed marked symptomatic improvement and was discharged three days later on oral contraceptive pills.

\section{Discussion}

Massive ovarian oedema (MOO) is a tumour-like enlargement 
of the ovary due to oedema fluid. It was first described in 1969 by Kalstone as a "massive, solid enlargement of the ovary associated with interstitial oedema without neoplastic changes" "and it is thought to be the result of incomplete torsion of the ovary interfering venous and lymphatic drainage but without causing necrosis. ${ }^{2}$ The great majority of cases are unilateral, affecting young women in the child-bearing period and can be easily mistaken for neoplasm. It has also been described during pregnancy and in postmenopausal women. MOO can occur as primary or secondary process. Primary MOO is seen in more than $85 \%$ of the reported cases and occurs when the ovary is not diseased and the torsion or twisting of the ovarian pedicle does not affect the arterial blood flow. Secondary massive ovarian oedema takes place in the setting of a diseased ovary such as an ovarian mass, malignancy, fibromatosis, polycystic ovary or secondary to the drugs used for ovulation induction. ${ }^{3}$ Common symptoms include pain, abdominal mass, menstrual irregularities, virilisation, precocious puberty and infertility.

Ovarian lymphatic vasculature is thought to play a vital role in fluid homeostasis and hormone trafficking, and alongside blood vasculature has been linked to several ovarian disorders such as polycystic ovary syndrome, ovarian hyperstimulation syndrome and massive ovarian oedema. The lymphatic dysfunction alters the normal luteinisation of the ovary which may lead to changes in normal ovarian steroidogenesis responsible for the clinical symptomatology of the MOO. Thus, masculinisation and precocious puberty could be common symptoms among women affected by this entity.

MOO is considered a rare disorder and unknown for most clinicians. A review of the literature proves that most patients are over treated with salpingo-oophorectomy on the basis that a primary ovarian neoplasm is suspected. In the review conducted by Praveen et al. 151 cases out of 177 were primary massive ovarian oedema and $88.7 \%$ of them did not have fertility-sparing surgery. ${ }^{4}$ Despite advanced radiological imaging MOO can easily over-diagnosed as ovarian neoplasm. However, the presence of peripherally embedded follicles may enable to differentiate between oedema and a neoplastic lesion. ${ }^{5} \mathrm{~A}$ twisted vascular pedicle between the enlarged ovary and the uterus may help to differentiate an adnexal torsion from MOO.

The differential diagnosis of MOO includes ovarian myxoma, fibromatosis and sclerosing stromal tumour (SST). Pure ovarian myxoma is sharply circumscribed, and normal ovarian tissue can be seen at its periphery, whereas massive ovarian edema often incorporates follicular structures at its periphery and spares the peripheral cortex. The SST has often a pseudolobular appearance with cellular areas separated by hyalinised connective tissue. Ovarian fibromatosis is a rare entity. Microscopy typically shows proliferation of spindle-shaped cells surrounding the normal follicular structure of the ovary. The superficial cortex is thickened and shows acellular bands of dense collagen. ${ }^{6} \mathrm{MOO}$ is a non-neoplastic disorder; therefore clinicians should consider this condition and take under consideration the age of patients presenting with this entity in order to preserve hormonal functions and their fertility.

\section{Conclusion}

Due to absence of pathognomonic clinical or radiological signs, when young women in the reproductive age group present with acute or chronic abdominal pain and a solid ovarian tumour-like mass, MOO should be suspected and conservative treatment proposed. Considering that medical management is not an option for this condition, wedge resection at the time of surgery and frozen section must be taken into consideration in order to preserve the fertility of these young patients.

\section{Acknowledgments}

None.

\section{Conflicts of interest}

The authors declare that they have no conflict of interest.

\section{References}

1. Kalstone CE, Jaffe RB, Abell MR. Massive edema of the ovary simulating fibroma. Obstet Gynecol. 1969;34(4):564-571.

2. Daboubi MK, Khreisat B. Massive ovarian oedema: literature review and case presentation. East Mediterr Health J. 2008;14(4):972-977.

3. Callen AL, Illangasekare T, Poder L. Massive ovarian edema, due to adjacent appendicitis. Emerg Radiol. 2017;24(2):215-218.

4. Praveen R, Pallavi V, Rajashekar K, et al. A clinical update on massive ovarian oedema - a pseudotumour? Ecancermedicalscience. 2013;7:318.

5. Tamai K, Koyama T, Saga T, et al. MR features of physiologic and benign conditions of the ovary. Eur Radiol. 2006;16(12):2700-2711.

6. Roth LM, Gaba AR, Cheng L. The pathogenesis of ovarian myxoma: a neoplasm sometimes arising from other ovarian stromal tumors. Int $J$ Gynecol Pathol. 2013;32(4):368-378. 